\title{
Clinical Outcomes of Proton Beam Therapy for Choroidal Melanoma at a Single Institute in Korea
}

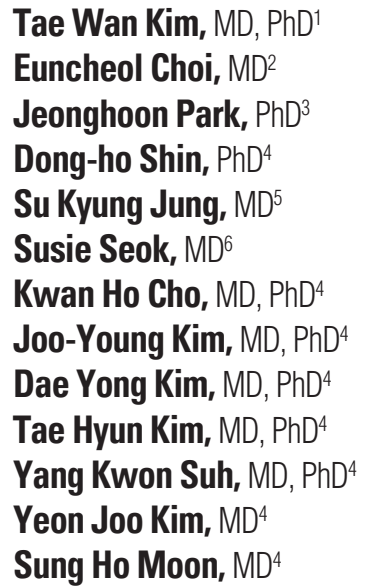

${ }^{1}$ Department of Ophthalmology, Seoul Metropolitan Government-Seoul National University Boramae Medical Center, Seoul National University College of Medicine, Seoul, ${ }^{2}$ Department of Radiation Oncology, Keimyung University Dongsan Medical Center, Keimyung University School of Medicine, Daegu, Korea, ${ }^{3}$ Department of Radiation Oncology, Baylor Scott \& White Health, Temple, TX, USA,

${ }^{4}$ Proton Therapy Center, Research Institute and Hospital, National Cancer Center, Goyang, ${ }^{5}$ Ophthalmology Clinic, Research Institute and Hospital, National Cancer Center, Goyang, ${ }^{6}$ Yonsei Jang's Eye Clinic, Seoul, Korea
Correspondence: Sung-Ho Moon, MD Proton Therapy Center, Research Institute and Hospital, National Cancer Center, 323 Ilsan-ro, Ilsandong-gu, Goyang 10408, Korea Tel: 82-31-920-1726

Fax: 82-31-920-0149

E-mail: shmoon@ncc.re.kr

Received February 9, 2017

Accepted April 17, 2017

Published Online April 19, 2017

*Tae Wan Kim and Euncheol Choi contributed equally to this work.

\section{Purpose}

This study retrospectively evaluated the clinical outcomes and complications of proton beam therapy (PBT) in a single institution in Korea and quantitatively analyzed the change in tumor volume after PBT using magnetic resonance imaging (MRI).

\section{Materials and Methods}

Twenty-four treatment-naïve patients who underwent PBT for choroidal melanoma between 2009 and 2015 were reviewed. Dose fractionation was 60-70 cobalt gray equivalents over 5 fractions. Orbital MRIs were taken at baseline and 3, 6, and 12 months after PBT and annually thereafter. The tumor volume was reconstructed and evaluated by stacking the tumor boundary in each thin-sliced axial T1-weighted image using MIM software.

\section{Results}

The median follow-up duration was 36.5 months (range, 9 to 82 months). One patient had suspicious local progression and two patients had distant metastasis. The 3-year local progression-free survival, distant metastasis-free survival, and overall survival rates were 95.8\%, 95.8\%, and 100\%, respectively. Five Common Terminology Criteria for Adverse Event ver. 4.03 grade 3-4 toxicities were observed in four patients (16.7\%), including one with neovascular glaucoma. The mean tumor volume at the baseline MRI was $0.565 \pm 0.084 \mathrm{~mL}$ (range, 0.074 to $1.610 \mathrm{~mL}$ ), and the ratios of the mean volume at 3,6 , and 12 months to that at baseline were $81.8 \%, 67.3 \%$, and $60.4 \%$, respectively.

\section{Conclusion}

The local control rate and complication profile after PBT in patients with choroidal melanoma in Korea were comparable with those reported in a previous PBT series. The change in tumor volume after PBT exhibited a gradual regression pattern on MRI.

\section{Key words}

Proton therapy, Choroid, Melanoma, Treatment outcome, Toxicity 


\section{Introduction}

Choroidal melanoma is the most common primary intraocular malignancy, with an incidence of 1.3-8.6 cases per one million individuals in Western countries [1,2]. Since eyeballconserving treatments such as proton beam therapy (PBT) or plaque therapy were revealed to be effective for the treatment of choroidal melanoma in the Collaborative Ocular Melanoma Study (COMS), enucleation is no longer a firstline standard treatment [3-7].

The annual incidence rate of choroidal melanoma in Asia was 0.25 cases per one million individuals, which was lower than that in Western countries, and thus the research on this neoplasm is limited $[8,9]$. The prognosis of choroidal melanoma is poorer in Asian populations, with epithelioid cell-type melanomas more common than in Caucasian populations $[9,10]$. However, two studies reported that the outcome of choroidal melanoma patients treated with plaque therapy was comparable to the outcome of those in a previous plaque therapy-treated Western series [11,12]. In addition, in a Japanese phase I/ II trial of carbon ion radiotherapy (RT) for choroidal melanoma, an excellent local control rate comparable to that of PBT was reported [13], and the updated long-term results were released recently [14].

Unlike other malignancies, there is skepticism regarding the use of biopsy at diagnosis or upon suspicion of local progression. In addition, delayed regression or pseudo-progression after eyeball-conserving treatment renders the decisionmaking associated with local tumor progression more difficult; inappropriate enucleation occurs in up to $40 \%$ of patients [15]. Although several investigators recently evaluated the kinetics of choroidal melanoma after eyeball-sparing treatment $[15,16]$, quantitative details regarding the change in tumor volume after eyeball-conserving treatment for choroidal melanoma are still limited.

In this retrospective study, we evaluated the clinical outcomes and toxicities of PBT at a single institution in Korea. We also quantitatively analyzed the change in tumor volume after PBT using orbital magnetic resonance image (MRI).

\section{Materials and Methods}

\section{Patients}

A total of 24 treatment-naïve patients were treated with PBT for choroidal melanoma between 2009 and 2015. As one of the eye conserving treatment modalities, PBT is not recommended to patients who are likely to undergo enucleation after treatment due to complication, especially neoavascular glaucoma (NVG) [17]. Thus, the selection criteria for PBT for choroidal melanoma were as follows: no distant metastasis, tumor diameter $\leq 24 \mathrm{~mm}$, tumor height $\leq 14 \mathrm{~mm}$, tumor volume $<30 \%$ of the ocular volume, and no full retinal detachment. These criteria are designed to minimize side effects and to achieve therapeutic effects. Similar criteria have been used in previous studies $[7,13,18,19]$. Ophthalmologic and systemic work-ups were performed to diagnose choroidal melanoma. The ophthalmologic work-up consisted of a slitlamp examination, indirect fundoscopy, ultrasonography (USG), fluorescein angiography, and orbit MRI followed by a systemic work-up of the liver using computed tomography (CT) or 18-fluorodeoxyglucose positron emission tomography.

\section{PBT planning}

After a pretreatment work-up, patients were transferred to a retina specialist for surgery to demarcate the tumor boundary using four tantalum markers. Orthogonal pairs of orbital X-ray radiographs were captured with the patient in the sitting position on a dedicated chair using a bite-block and facial mask while maintaining a straight gaze during simulation. The tantalum clips in the X-ray images were localized and analyzed in VIVA systems (Varian Medical Systems, Palo Alto, CA). Then, eyeball biometry data were acquired from the ophthalmologist, and the localized coordinates were used to create an eyeball model for the patient using the ocular treatment planning system Varian EYEPLAN V1.0 (Varian Medical Systems). Thin-slice (0.625 mm) planning CT images were also taken with the patient in the supine position gazing straight forward. The external contour of the bilateral eyeballs, tantalum clips, gross tumor volume, and intraocular structures including the lenses and optic nerves were contoured on the planning CT to create three-dimensional reconstructed images for each patient. The target volume delineation was modeled using the analytic function in EYEPLAN to ensure that it closely matched the panoramic details in fundoscopic images, pretreatment MRI, and reconstructed thin-slice CT images. Static tantalum clip positions were reconstructed on the eye globe as close to the simulation positions as possible. In the planning stage, a gaze direction was selected along the optimal treatment setup to deliver the proton beam and minimize the dose to the optic disc, macula, anterior structures (lens, cornea, and anterior chamber), lacrimal gland, and lids. In the verification stage, the clip positions of the treatment gaze angle were matched with those acquired from the EYEPLAN system. A brass aperture was then manufactured with a 3-mm margin around the tumor. The dose prescription was 60-70 cobalt gray equivalents (CGE) in five fractions. Six early patients 

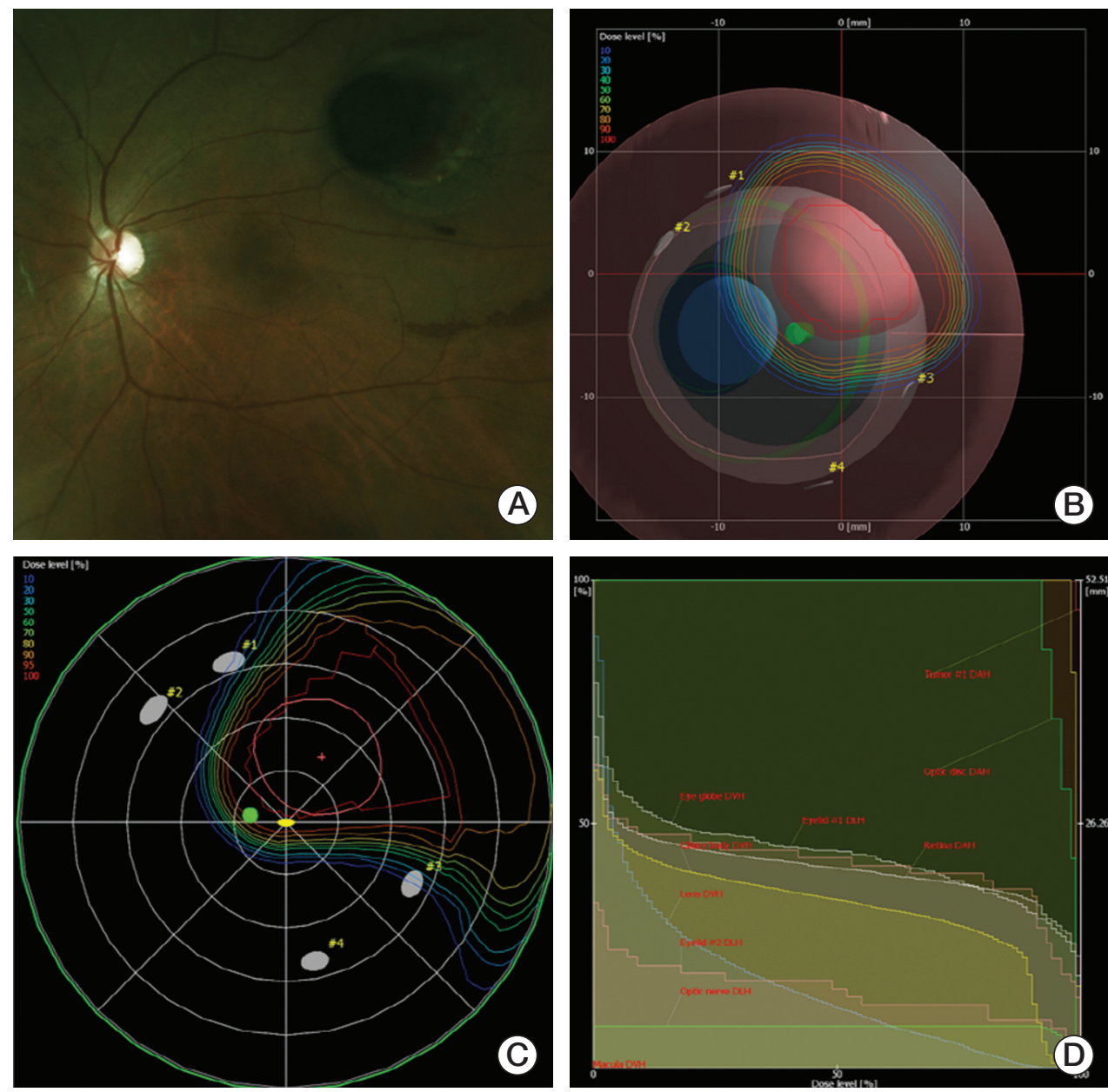

Fig. 1. A case of proton beam therapy (PBT) planning in eyeball model of a patient with choroidal melanoma. (A) Fundoscopic finding suggested the tumor located near 2 disc diameter from macula. (B) Beams-eyed view. (C) Dose distribution of PBT in fundus view. (D) Dose volume histogram.

were treated with 60 CGE in five fractions, and 70 CGE in five fractions scheme was adopted for subsequent patients. A case of PBT planning in eyeball model of a patient with choroidal melanoma is shown in Fig. 1.

\section{PBT delivery}

At our institution, eye treatment is performed in a fixed beam room using an energy of $230 \mathrm{MeV}$ (Proteus-235, IBA, Louvain-la-Neuve, Belgium). The simulation setup is reproduced using orthogonal X-ray systems with clip positions with $<0.3 \mathrm{~mm}$ deviations. The treatment beam was irradiated in single-scattering mode with the eye treatment beam current modulation (BCM) option. In the BCM option, the lateral penumbra was $2 \mathrm{~mm}$ and the distal penumbra was $5 \mathrm{~mm}$. The maximum beam range and field radius were
$3.5 \mathrm{~cm}$ and $3 \mathrm{~cm}$, respectively. The deviation of the beam range and width were within $0.1 \mathrm{~mm}$ on the multi-layer ion chamber during the quality assurance procedure.

Our in-house automatic beam gating system utilizes a method of image pattern matching. An initial gaze setup image was taken using the in-room CCD camera. Eye movement was monitored and analyzed in real-time during beam irradiation. If the patient's eye movement exceeded the tolerance (initially set at $1.0 \mathrm{~mm}$ ), the proton beam delivery was stopped within 10 milliseconds. This system utilizes the same gating signals and beam control circuits with the Real-time Position Management system (Varian Medical Systems). In previous study [20], the detection accuracy of this system was $0.1 \mathrm{~mm}$, and the mean eye movement was $0.44-0.86 \mathrm{~mm}$ for three patients. More detailed descriptions of the beam gating system can be found in the study mentioned above [20]. 
Table 1. Patients' characteristics

\begin{tabular}{|c|c|}
\hline Characteristic & No. of patients $(\%)$ \\
\hline \multicolumn{2}{|l|}{ Age (yr) } \\
\hline Median & 62 \\
\hline Mean (range) & $62.58(44-85)$ \\
\hline \multicolumn{2}{|l|}{ Sex } \\
\hline Male & $14(58.3)$ \\
\hline Female & $10(41.7)$ \\
\hline \multicolumn{2}{|l|}{ Initial symptom } \\
\hline Visual acuity decreasing & $9(37.5)$ \\
\hline Ocular discomfort & $4(16.7)$ \\
\hline Vitreous floaters & $5(20.8)$ \\
\hline Visual field defect & $3(12.5)$ \\
\hline Incidental finding on regular check-ups & $2(8.3)$ \\
\hline $\begin{array}{l}\text { Both decreased of visual acuity and } \\
\text { visual field defect }\end{array}$ & $1(4.2)$ \\
\hline \multicolumn{2}{|l|}{ ECOG } \\
\hline 0 & $22(91.7)$ \\
\hline 1 & $2(8.3)$ \\
\hline \multicolumn{2}{|l|}{ Nevus } \\
\hline Negative & $24(100)$ \\
\hline Positive & 0 \\
\hline \multicolumn{2}{|l|}{ Involved eye } \\
\hline Right eye & $7(29.2)$ \\
\hline Left eye & $17(70.8)$ \\
\hline \multicolumn{2}{|l|}{ Location } \\
\hline Pre-equatorial & $5(20.8)$ \\
\hline Equatorial & $7(29.2)$ \\
\hline Retro-equatorial & $12(50.0)$ \\
\hline \multicolumn{2}{|l|}{ Growth pattern } \\
\hline Dome & $15(62.5)$ \\
\hline Mushroom & $7(29.2)$ \\
\hline Diffuse & $2(8.3)$ \\
\hline Basal tumor diameter, median (range, mm) & $11(4.4-16)$ \\
\hline Tumor thickness, median (range, mm) & $8(2-13)$ \\
\hline \multicolumn{2}{|l|}{ Ciliary body involvement } \\
\hline Negative & $22(91.7)$ \\
\hline Positive & $2(8.3)$ \\
\hline \multicolumn{2}{|l|}{ Extraocular spread } \\
\hline Negative & $24(100)$ \\
\hline Positive & 0 \\
\hline \multicolumn{2}{|l|}{ Optic disc involvement } \\
\hline Negative & $24(100)$ \\
\hline Positive & 0 \\
\hline \multicolumn{2}{|l|}{$\begin{array}{l}\text { Distance from posterior tumor border } \\
\text { to fovea (ODD) }\end{array}$} \\
\hline$\leq 2$ & $7(29.2)$ \\
\hline$>2$ & $17(70.8)$ \\
\hline \multicolumn{2}{|l|}{$\begin{array}{l}\text { Distance from posterior tumor border } \\
\text { to optic disc (ODD) }\end{array}$} \\
\hline$\leq 2$ & $2(8.3)$ \\
\hline$>2$ & $22(91.7)$ \\
\hline
\end{tabular}

(Continued)
Table 1. Continued

\begin{tabular}{lc} 
Characteristic & No. of patients (\%) \\
Initial retinal detachment & \\
$\quad$ Negative & $16(66.7)$ \\
$\quad$ Positive & $8(33.3)$ \\
Intraocular hemorrhage & \\
$\quad$ Negative & $16(66.7)$ \\
\hline Positive & $8(33.3)$ \\
\hline AJCC staging & \\
I & $6(25.0)$ \\
II & $16(66.7)$ \\
III & $2(8.3)$ \\
IV & 0 \\
\hline Tumor size category ${ }^{\text {b) }}$ & \\
\hline Small & $3(12.5)$ \\
\hline Medium & $5(20.8)$ \\
\hline Large & $15(62.5)$ \\
Extra large & $1(4.2)$ \\
\hline
\end{tabular}

ECOG, Eastern Cooperative Oncology Group; ODD, optic

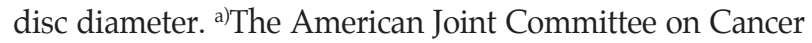
(AJCC) classification seventh edition, by Egger et al. [7]; small: diameter $\leq 10 \mathrm{~mm}$ and thickness $\leq 3 \mathrm{~mm}$, medium: $10 \mathrm{~mm}<$ diameter $\leq 15 \mathrm{~mm}$ and/ or $3 \mathrm{~mm}<$ thickness $\leq 5$ $\mathrm{mm}$, large: $15 \mathrm{~mm}<$ diameter $\leq 20 \mathrm{~mm}$ and/or $5 \mathrm{~mm}$ $<$ thickness $\leq 10 \mathrm{~mm}$, extra large: diameter $>20 \mathrm{~mm}$ and / or thickness $>10 \mathrm{~mm}$.

\section{Follow-up evaluation}

Patients were routinely followed up after 1 month for acute reactions and every 3 months thereafter after PBT. Ophthalmologic examinations were performed at every visit. Followup orbital MRIs were performed at 3, 6, and 12 months after PBT and annually thereafter for most patients. Complications were graded according to the Common Terminology Criteria for Adverse Events ver. 4.03. Survival curves were generated using the Kaplan-Meier method. The endpoints were local progression-free survival, distant metastasis-free survival, and overall survival. Local progression and distant metastasis were defined as an increase in tumor height of $15 \%$ on follow-up USG, according to the definition of recurrence determined by the COMS [21], and confirmed extraocular disease on MRI or another systemic imaging procedure at follow up, respectively.

\section{Volumetric evaluation}

In this study, volumetric evaluations were performed using MIM software (MIM software Inc., Cleveland, $\mathrm{OH}$ ). 
T2-weighted and contrast-enhanced T1-weighted images were imported into the MIM software. Then, a radiation oncologist contoured the tumor boundary on each thin-slice axial contrast-enhanced T1-weighted image for each patient. Another radiation oncologist reviewed and confirmed the tumor contouring and shape. Retinal detachment was excluded from the tumor volume. Volumetric changes in the tumor were observed by MRI at 3, 6, and 12 months and at the last follow-up. In addition, two groups of patients categorized by tumor volume (small and medium vs. large and extra large), as described by Egger et al. [7] (Table 1), were analyzed to determine the difference in regression according to tumor volume. Mann-Whitney tests were used for subgroup analyses.

\section{Results}

The patients' characteristics are summarized in Table 1 . The median patient age was 62 years (range, 44 to 85 years). There were similar numbers of male and female patients. The most common chief complaint was a decrease in visual acuity (VA; nine patients, $37.5 \%$ ), followed by a floater (five patients, $20.8 \%$ ) and ocular discomfort (four patients, 16.7\%). The median basal tumor diameter and thickness were $11 \mathrm{~mm}$ (range, 4.4 to $16 \mathrm{~mm}$ ) and $8 \mathrm{~mm}$ (range, 2 to $13 \mathrm{~mm}$ ), respectively. The ciliary body was involved in two patients $(8.3 \%)$. Extraocular spreads or optic disc involvements were not found in any of the baseline work-ups. An optic disc distance (ODD) of $<2$ from the tumor to the foveal center was seen in seven patients $(29.2 \%)$. An ODD of $<2$ from the tumor to the optic disc was seen in two patients $(8.3 \%)$. Localized retinal detachment was observed in eight patients (33.3\%). Vitreous hemorrhages were detected in eight patients $(33.3 \%)$. There were no lymph node metastases or distant metastases.

The median follow-up duration after PBT was 36.5 months (range, 9 to 82 months). Eight patients exhibited localized retinal detachment, of whom seven showed complete resolution and one showed partial resolution. Three patients exhibited disease progression, one of whom had local progression and two of whom developed distant metastases. In the patient with local progression, findings of consecutive submillimeter increases on serial USG, CT, and MRI with floating cells on serial fundoscopy led to suspicions of local progression. The patient was observed closely because of an inconsistency with the COMS definition of local progression. Then, local excision and plaque brachytherapy (54.5 Gy) were performed as salvage treatments at another hospital 18 months after PBT. In the patient's pathologic report of the surgical specimen, mitotic cells were found in less than 1/40 of the high-power

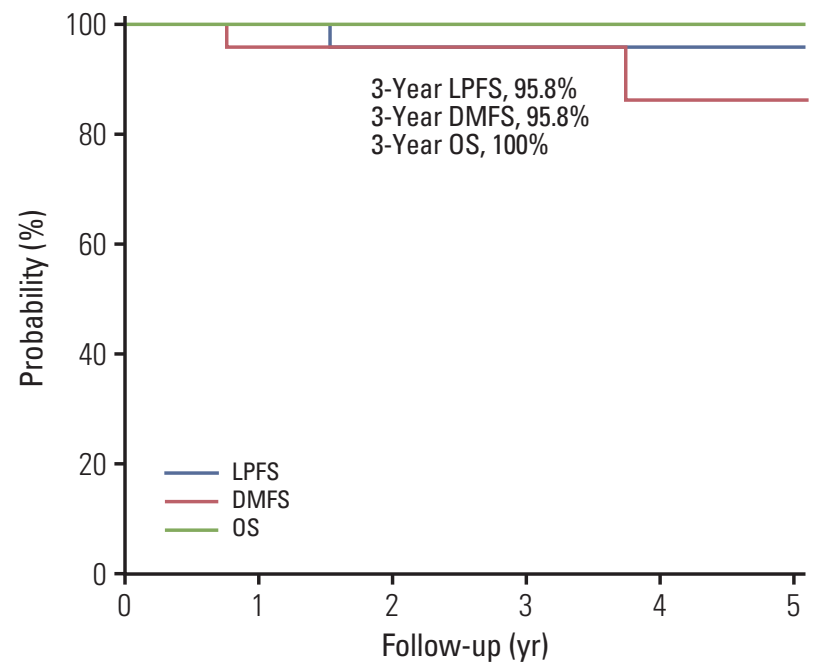

Fig. 2. Survival curves. LPFS, local progression-free survival; DMFS, distant metastasis-free survival; OS, overall survival.

field. Currently, after 60 months of follow-up, there is still no evidence of disease. There were two patients with distant metastasis. In a patient, a single liver metastasis was found during systemic work-up followed after detection of mass-like lesion adjacent to extraocular muscle in orbital magnetic resonanc images at 47 months after diagnosis. It was recommended that the patient undergo a tumor-directed biopsy of the orbital apex lesion, but she refused because of fear of vision loss. The patient did not want to continue further treatment because of the low life expectancy associated with liver metastasis. The patient has survived 18 months since her diagnosis of distant metastasis. In another patient with distant metastasis, a single liver metastatic lesion was detected by CT performed at 11 months after diagnosis.

Chemotherapy was recommended. There was no follow up after the diagnosis of distant metastasis. The 3-year local progression free survival, distant metastasis-free survival, and overall survival rates were $95.8 \%, 95.8 \%$, and $100 \%$, respectively (Fig. 2).

The treatment-related toxicities are listed in Table 2. Most of toxicities were mild-to-moderate, but five severe toxicities were noted in four patients. Of these patients, one was enucleated due to late grade 4 NVG. Another patient with acute grade 4 vitreous hemorrhage underwent vitrectomy. The third patient with late grade 3 posterior synechia had increasing intraocular pressure and was treated using laser iridotomy. The fourth patient with grade 3 NVG underwent an intravitreal bevacizumab injection. There was no evidence of recurrence in these patients with severe toxicities. The eyes were all 
Table 2. Acute and late toxicities by Common Terminology Criteria for Adverse Event ver. 4.03

\begin{tabular}{|c|c|c|c|c|}
\hline & \multicolumn{2}{|c|}{ Acute toxicity } & \multicolumn{2}{|c|}{ Late toxicity } \\
\hline & Grade $<3$ & Grade $\geq 3$ & Grade $<3$ & Grade $\geq 3$ \\
\hline Erythroderma & $7(29.2)$ & - & $2(8.3)$ & - \\
\hline Blurred vision & - & - & $1(4.2)$ & - \\
\hline Retinal detachment & $2(8.4)$ & - & - & - \\
\hline Vitreous hemorrhage & - & $1(4.2)$ & - & - \\
\hline Cataract & - & $1(4.2)$ & - & - \\
\hline Glaucoma & - & - & $5(20.8)$ & $2(8.4)$ \\
\hline Retinopathy & - & - & $1(4.2)$ & - \\
\hline Eye disoders-other, specifya) & $1(4.2)$ & - & $1(4.2)$ & $1(4.2)$ \\
\hline
\end{tabular}

Values are presented as number (\%). a)Posterior synechia.

Table 3. Summary of dose volume histogram parameters of patients with acute or late grade $\geq 3$ toxicity

\begin{tabular}{|c|c|c|c|c|c|c|}
\hline Patient & GTV (mL) & V30_retina $(\%)$ & V30_cb ${ }^{\text {a) }}(\%)$ & V30_mac ${ }^{\mathrm{b})}(\%)$ & V30_OD ${ }^{c}(\%)$ & Toxicity \\
\hline 1 & 1.95 & 50.80 & 45.00 & 0.00 & 30.00 & Cataract G4, NVG G4 \\
\hline 2 & 0.74 & 33.40 & 39.00 & 0.00 & 0.00 & Vitreous hemorrhage G4 \\
\hline 3 & 1.41 & 53.20 & 47.10 & 54.70 & 71.26 & Posterior synechia G3 \\
\hline 4 & 1.20 & 49.00 & 38.80 & 100.00 & 57.20 & Posterior synechia G3 \\
\hline $\begin{array}{l}\text { With grade } \\
\geq 3 \text { toxicity (mean) }\end{array}$ & 1.32 & 46.60 & 42.48 & 38.68 & 39.62 & \\
\hline $\begin{array}{l}\text { Without grade } \\
\geq 3 \text { toxicity (mean) }\end{array}$ & 0.90 & 38.28 & 31.65 & 49.45 & 55.72 & \\
\hline p-value ${ }^{\mathrm{d})}$ & 0.203 & 0.167 & 0.006 & 0.703 & 0.434 & \\
\hline
\end{tabular}

GTV, gross tumor volume; NVG, neovascular glaucoma. ${ }^{\text {a) } V o l u m e ~ o f ~ c i l i a r y ~ b o d y ~ r e c e i v i n g ~} 30$ cobalt gray equivalent (CGE),

${ }^{b}$ Volume of mac receiving 30 CGE, c)Volume of optic disc receiving 30 CGE, d)By independent $t$ test.

Table 4. Changes of visual acuity after proton beam therapy

\begin{tabular}{lrrrc} 
Visual acuity & Baseline & 3 Months & $\mathbf{6}$ Months & Final \\
$20 / 40-20 / 20$ & $10(41.7)$ & $5(20.8)$ & $4(16.7)$ & $4(16.7)$ \\
$20 / 100-20 / 40$ & $5(20.8)$ & $6(25.0)$ & $5(20.8)$ & $4(16.7)$ \\
$20 / 200-20 / 100$ & $4(16.7)$ & $1(4.2)$ & $2(8.4)$ & 0 \\
$\leq 20 / 200$ & $5(20.8)$ & $12(50.0)$ & $13(54.1)$ & $16(66.6)$ \\
\hline
\end{tabular}

Values are presented as number $(\%)$.

preserved, except in one patient who developed NVG and was treated with enucleation. Therefore, the 3-year enucleationfree rate was $95.8 \%$. Dose volume histogram parameters of patients with acute or late grade $\geq 3$ toxicity were summarized in Table 3.

The percentage of patients with $>20 / 40$ Snellen VA was
$41.7 \%$ at baseline, which decreased to $16.7 \%$ at the final follow-up. A total of $58.3 \%$ and $45.8 \%$ of patients lost at least three or at least five lines of Snellen VA, respectively. The aspect of visual change is shown in Table 4 . Six patients maintained their VA. Five patients had a small-sized tumor without involvement of the optic disc. One patient with a large tumor 

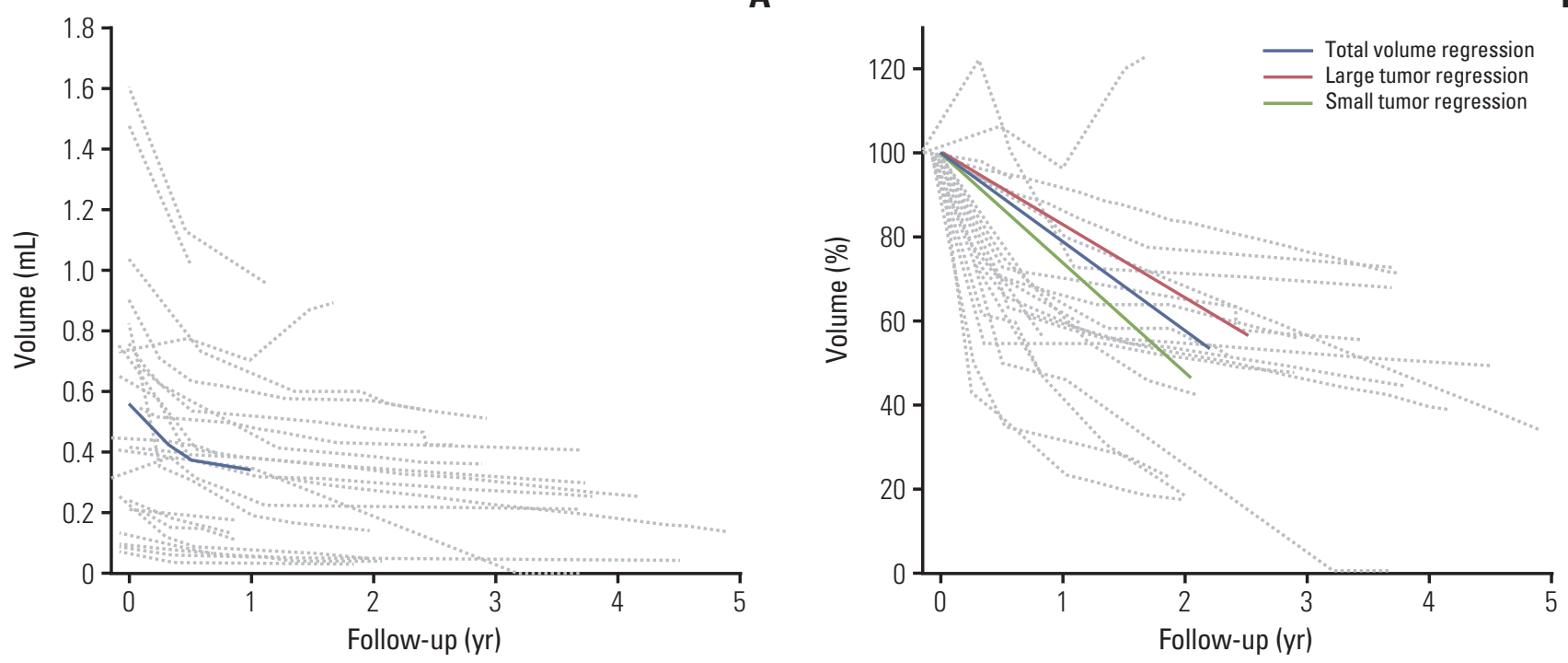

Fig. 3. The volumetric change in the choroidal melanoma of each patient. Solid lines show the volumetric changes in recurrent tumors. (A) Absolute changes in tumor volume. Thick solid lines show the changes in the mean volume 12 months after proton beam therapy. (B) The relative changes in tumor volume. The thick solid line shows the regression of the relative volume. The thick and short-dashed lines represent the regression lines of patients with large tumors. The thick and longdashed lines represent the regression lines of patients with small tumors.

maintained VA because of the large distance between the tumor and the optic disc.

Apart from one patient treated with enucleation due to NVG, volumetric analysis was performed in the remaining 23 patients. All patients started PBT within 2 months of the initial MRI. The mean volume on the initial MRI was $0.565 \pm 0.084 \mathrm{~mL}$ (range, 0.074 to $1.610 \mathrm{~mL}$ ). The ratios of the mean volume at 3 , 6 , or 12 months to that at baseline were $81.8 \%, 67.3 \%$, and $60.4 \%$, respectively. The ratio of the mean volume reduction on the last follow-up MRI to that on the baseline MRI was $47.6 \%$. The tumor volume exhibited a gradual regression pattern in all patients, except one who experienced local progression after PBT. The volumetric changes in each patient are depicted in Fig. 3. Regression lines of the large and small tumors are also shown, but there was no statistically significant difference in volumetric regression ( $p>0.99)$. When comparing the groups of more than $50 \%$ mean volume reduction vs. not, no statistical difference was found in terms of 3-year local control rate $(100 \%$ vs. $88.9 \%, \mathrm{p}=0.269)$ and 3-year distant metastasis free survival rate $(100 \%$ vs. $88.9 \%, \mathrm{p}=0.130)$.

\section{Discussion}

The objectives of this study were to evaluate the survival, visual outcomes, and complications of patients undergoing PBT in a single institution and quantitatively analyze the changes in tumor volume using MRI volumetry. As described above, enucleation is no longer considered the initial treatment for choroidal melanoma, except when the tumor extends widely into the nearby sclera or extensively involves the ocular volume. Plaque therapy using cobalt-60, ruthenium-106, and iodine-125 has been commonly used worldwide as an eyeball-sparing treatment for choroidal melanoma, and the local control rates were slightly higher compared with those of particle beam treatment [22-24]. Eye treatment using dedicated beam line PBT has advantages over stereotactic radiosurgery because of sharp lateral and distal penumbra; however, there are problems regarding access to PBT facilities. While our PBT center, as the first in Korea, has treated patients since March 2007, PBT was first used as a dedicated eye treatment in 2009. Although little research has investigated choroidal melanoma in Asia, a retrospective study evaluated ruthenium-106 ( $\left.{ }^{106} \mathrm{Ru}\right)$ plaque brachytherapy in Korea, and the serial short- and long-term updated results of a phase I/II Japanese dose-escalation study of carbon ion RT for choroidal melanoma were 
reported recently $[13,14]$. The outcome results of ${ }^{106} \mathrm{Ru}$ plaque brachytherapy were similar to those in Caucasian patients; however, the 5-year local recurrence rate and the incidence of enucleation were relatively high $(16.9 \%$ and $25.4 \%$, respectively). The authors suggested that this might be because the radiation dose was insufficient to cover large tumors [11]. In contrast, the 5-year local control and eye retention rates were $92.8 \%$ and $92.8 \%$, respectively, in a phase I/ II carbon ion RT study that achieved historical results comparable to those of PBT, although patients with relatively large tumors or tumors in an unfavorable location close to the optic disc were included [14].

Controlling eye movement effectively is one of the most important steps in the delivery of PBT, because patients tend to struggle concentrating their eyes on the fixation light point during the period of beam delivery. Even if the tumor location shifts by a few millimeters, the dose delivered can vary by tens of percentages. Thus, a precise and reliable technique for eye-tracking and an automatic beam gating system would be beneficial. In the current study, an in-house motion-based gated PBT system was used in all patients. A variety of tests for accuracy and safety of eye tracking and gating were done before application to treatment and patient data for eye movement during treatment were acquired for the early 3 patients with PBT [20]. For the first six patients treated in the current study, the dose fractionation was set at 6,000 CGE in five fractions as a learning curve to ensure that the treatment was safe. Thereafter, the fractional radiation dose was safely escalated from 1,200 to 1,400 CGE. The only case of local progression was observed in a patient who received the 1,200 CGE of fractional dose; therefore, it would be difficult to compare the difference in outcomes between 1,200 CGE and 1,400 CGE groups because of the small number of patients treated. Furthermore, no differences in tumor regrowth or metastasis between 1,000 and 1,400 CGE have been reported $[5,6,18,25]$. Although only a small number of patients were included, the current data revealed that PBT achieved an excellent local control rate and eyeball-sparing capability in an Asian population with choroidal melanoma. The results were comparable to those of a larger series of Western PBT patients [5-7,26-29] and carbon ion RT studies [29].

We used Common Terminology Criteria for Adverse Event (CTCAE) ver. 4.03 to evaluate the toxicity after treatment in our study, but the interpretation of 3-4 grade toxicity in choroidal melanoma would be different from in other solid organ malignancies, given that the most severe treatment-related toxicity is regarded as enucleation, not death. Because it is difficult to evaluate the toxicity after RT, with various tumor-related and treatment parameters to be considered together, there is no established scoring system in choroidal melanoma yet [30]. As suggested in many other studies, NVG is one of the most troublesome complications associated with the treatment of choroidal melanoma and the most common cause of enucleation $[5,6,21,26,27,31]$. Some investigators suggested the presence of rubeosis iridis as a marker of severe ocular damage, which can result in subsequent enucleation [32]. Increased intraocular pressure developed in seven patients $(29.2 \%)$ in the current study, but only two $(8.4 \%)$ experienced severe NVG, superior to other PBT reports $[5,6,18,19]$. All of five acute or late grade $\geq 3$ toxicities developed in four patients with rather huge-sized choroidal melanoma with $1.3 \mathrm{~mL}$ of average gross tumor volume (vs. $0.9 \mathrm{~mL}$ in others). One patient underwent enucleation, but the other patients are stable and managing their own lives relatively well after minor interventional procedures. Of note, the incidence of rubeosis iridis and NVG is $29.2 \%$ in our study was comparable to other studies of PBT with 25\%-45\% $[5,6,18,19,32]$. Other complication profiles were also comparable, but long-term follow up is needed. Although the precise relationship between irradiation to anterior chamber and the development of NVG is not clear, the amount of lens or anterior chamber volume, or ciliary body volume in eye model is considered the important parameter in PBT planning [27]. Lens volume or ciliary body receiving at least 30 CGE was suggested as an independent prognostic factor for enucleation in a retrospective study, but there is also no consensus guideline about this.

The strongest predictor of visual prognosis is known to be the location of tumor relative to fovea and optic disc [33-35]. Although the anterior chamber dose and optic disc or fovea dose can directly affect the risk of NVG and the vision loss in such a large dose per fraction in RT for choroidal melanoma, which is a basic priniciple of radiation oncology, there is no dose constraint guideline for anterior chamber, optic disc and fovea in the PBT planning. Avoiding the risk of NVG with decreasing the dose to anterior chamber sometimes significantly increases optic disc or fovea dose. In various studies assessing visual outcomes after treatment $[6,26,36]$, the visual loss of more than three and five lines of Snellen VA occurred in $\sim 45 \%$ and $~ 25 \%$ of patients, respectively. The visual outcomes in the current study seem slightly worse than those in previous studies. However, it should be considered that the most important predictor of visual outcome after RT would be more closely related with the location of the tumor relative to the optic disc and fovea rather than treatment factors. Also, the relative priority of preserving the eyeball should be considered during the planning stage of PBT, particularly in cases of conflict when weighing the risks between NVG and VA reduction, i.e., the anterior chamber versus the optic disc or fovea. Therefore, a meaningful analysis of the visual outcome after PBT might also be difficult in the present study.

The volumetric evaluation of choroidal melanoma was also an issue evaluated in the current study. A gradual pat- 
tern of short-term tumor regression was also found, similar to the findings of Russo et al. [16]. However, there was no significant difference in volume regression according to tumor size. There also was no significant difference between the groups of more than $50 \%$ versus not in terms of clinical outcome of local control rate and distant metastasis free survival in our study. Considering that the events of any disease progression were relatively rare in our study and especially, distant metastasis in choroidal melanoma might be quite a late process of more that 15-20 years [37], a study of longer term follow-up with a large number of patients could answer the question of the association between the degree of regression after proton therapy and the survival outcome. The current COMS definition of tumor recurrence is based on the two-dimensional length of the tumor determined by USG [15], and the use of orbit MRI is also expected to be helpful during the short-term follow-up period.

There were some limitations to the current study. First, it was a retrospective study involving a small number of patients, and the relatively short follow-up period was not sufficient for the natural course of choroidal melanoma after treatment. Nevertheless, the results are meaningful, because they provide rare evidence of PBT for choroidal melanoma in Asia and reveal similar outcomes to those performed in Western countries.

In conclusion, PBT achieved a local control rate in Korean patients with choroidal melanoma that was comparable with those in previously reported PBT series. PBT also had an acceptable toxicity, comparable with those in Western countries.

\section{Conflicts of Interest}

Conflict of interest relevant to this article was not reported.

\section{Acknowledgments}

This study was supported by National Cancer Center Grants (1610590).

\section{References}

1. Virgili G, Gatta G, Ciccolallo L, Capocaccia R, Biggeri A, Crocetti E, et al. Incidence of uveal melanoma in Europe. Ophthalmology. 2007;114:2309-15.

2. Singh AD, Turell ME, Topham AK. Uveal melanoma: trends in incidence, treatment, and survival. Ophthalmology. 2011; 118:1881-5.

3. Diener-West M, Reynolds SM, Agugliaro DJ, Caldwell R, Cumming K, Earle JD, et al. Screening for metastasis from choroidal melanoma: the Collaborative Ocular Melanoma Study Group Report 23. J Clin Oncol. 2004;22:2438-44.

4. Shields JA, Shields CL. Management of posterior uveal melanoma: past, present, and future: the 2014 Charles L. Schepens lecture. Ophthalmology. 2015;122:414-28.

5. Gragoudas ES, Lane AM, Munzenrider J, Egan KM, Li W. Long-term risk of local failure after proton therapy for choroidal / ciliary body melanoma. Trans Am Ophthalmol Soc. 2002;100:43-8.

6. Gragoudas E, Li W, Goitein M, Lane AM, Munzenrider JE, Egan KM. Evidence-based estimates of outcome in patients irradiated for intraocular melanoma. Arch Ophthalmol. 2002; 120:1665-71.

7. Egger E, Schalenbourg A, Zografos L, Bercher L, Boehringer $\mathrm{T}$, Chamot L, et al. Maximizing local tumor control and survival after proton beam radiotherapy of uveal melanoma. Int J Radiat Oncol Biol Phys. 2001;51:138-47.

8. Kaneko A. Malignant ophthalmic tumors. Nihon Rinsho. 1993;51 Suppl:1013-20.

9. Kuo PK, Puliafito CA, Wang KM, Liu HS, Wu BF. Uveal melanoma in China. Int Ophthalmol Clin. 1982;22:57-71.

10. Sakamoto T, Sakamoto M, Yoshikawa H, Hata Y, Ishibashi T, Ohnishi $Y$, et al. Histologic findings and prognosis of uveal malignant melanoma in japanese patients. Am J Ophthalmol. 1996;121:276-83.

11. Kwon HJ, Ko JS, Kim M, Lee CS, Lee SC. Prognosis of choroidal melanoma and the result of ruthenium brachytherapy combined with transpupillary thermotherapy in Korean patients. Br J Ophthalmol. 2013;97:653-8.

12. Shah PK, Selvaraj U, Narendran V, Guhan P, Saxena SK, Dash A. Indigenous (125)I brachytherapy source for the management of intraocular melanomas in India. Cancer Biother Radiopharm. 2013;28:21-8.

13. Tsuji $H$, Ishikawa H, Yanagi T, Hirasawa N, Kamada T, Mizoe JE, et al. Carbon-ion radiotherapy for locally advanced or unfavorably located choroidal melanoma: a Phase I/II doseescalation study. Int J Radiat Oncol Biol Phys. 2007;67:857-62.

14. Toyama S, Tsuji H, Mizoguchi N, Nomiya T, Kamada T, Tokumaru $S$, et al. Long-term results of carbon ion radiation therapy for locally advanced or unfavorably located choroidal melanoma: usefulness of CT-based 2-port orthogonal therapy for reducing the incidence of neovascular glaucoma. Int J Radiat Oncol Biol Phys. 2013;86:270-6.

15. Maschi C, Thariat J, Herault J, Caujolle JP. Tumour response in uveal melanomas treated with proton beam therapy. Clin Oncol (R Coll Radiol). 2016;28:198-203.

16. Russo A, Mariotti C, Longo A, Foti PV, Avitabile T, Uva MG, et al. Diffusion-weighted magnetic resonance imaging and 
ultrasound evaluation of choroidal melanomas after protonbeam therapy. Radiol Med. 2015;120:634-40.

17. Egan KM, Gragoudas ES, Seddon JM, Glynn RJ, Munzenreider JE, Goitein M, et al. The risk of enucleation after proton beam irradiation of uveal melanoma. Ophthalmology. 1989;96: 1377-82.

18. Gragoudas ES. Proton beam irradiation of uveal melanomas: the first 30 years. The Weisenfeld Lecture. Invest Ophthalmol Vis Sci. 2006;47:4666-73.

19. Gragoudas ES, Marie Lane A. Uveal melanoma: proton beam irradiation. Ophthalmol Clin North Am. 2005;18:111-8.

20. Shin D, Yoo SH, Moon SH, Yoon M, Lee SB, Park SY. Eye tracking and gating system for proton therapy of orbital tumors. Med Phys. 2012;39:4265-73.

21. Jampol LM, Moy CS, Murray TG, Reynolds SM, Albert DM, Schachat AP, et al. The COMS randomized trial of iodine 125 brachytherapy for choroidal melanoma: IV. Local treatment failure and enucleation in the first 5 years after brachy therapy. COMS report no. 19. Ophthalmology. 2002;109:2197-206.

22. Shields JA, Augsburger JJ, Brady LW, Day JL. Cobalt plaque therapy of posterior uveal melanomas. Ophthalmology. 1982; 89:1201-7.

23. Lommatzsch PK. Beta-Irradiation of choroidal melanoma with 106Ru/106Rh applicators. 16 Years' experience. Arch Ophthalmol. 1983;101:713-7.

24. Packer S, Rotman M. Radiotherapy of choroidal melanoma with iodine-125. Ophthalmology. 1980;87:582-90.

25. Gragoudas ES, Egan KM, Saornil MA, Walsh SM, Albert DM, Seddon JM. The time course of irradiation changes in proton beam-treated uveal melanomas. Ophthalmology. 1993;100: 1555-9.

26. Sikuade MJ, Salvi S, Rundle PA, Errington DG, Kacperek A, Rennie IG. Outcomes of treatment with stereotactic radiosurgery or proton beam therapy for choroidal melanoma. Eye (Lond). 2015;29:1194-8.

27. Mishra KK, Quivey JM, Daftari IK, Weinberg V, Cole TB, Patel $\mathrm{K}$, et al. Long-term results of the UCSF-LBNL randomized trial: charged particle with helium ion versus Iodine-125 plaque therapy for choroidal and ciliary body melanoma. Int J Radiat Oncol Biol Phys. 2015;92:376-83.
28. Browne AW, Dandapani SV, Jennelle R, Stevanovic M, Lee TC, Murphree AL, et al. Outcomes of medium choroidal melanomas treated with ruthenium brachytherapy guided by three-dimensional pretreatment modeling. Brachytherapy. 2015;14:718-25.

29. Wang Z, Nabhan M, Schild SE, Stafford SL, Petersen IA, Foote $\mathrm{RL}$, et al. Charged particle radiation therapy for uveal melanoma: a systematic review and meta-analysis. Int J Radiat Oncol Biol Phys. 2013;86:18-26.

30. Hocht S, Bechrakis NE, Nausner M, Kreusel KM, Kluge H, Heese J, et al. Proton therapy of uveal melanomas in Berlin: 5 years of experience at the Hahn-Meitner Institute. Strahlenther Onkol. 2004;180:419-24.

31. Summanen P, Immonen I, Kivela T, Tommila P, Heikkonen J, Tarkkanen A. Radiation related complications after ruthenium plaque radiotherapy of uveal melanoma. Br J Ophthalmol. 1996;80:732-9.

32. Foss AJ, Whelehan I, Hungerford JL, Anderson DF, Errington $\mathrm{RD}$, Kacperek A, et al. Predictive factors for the development of rubeosis following proton beam radiotherapy for uveal melanoma. Br J Ophthalmol. 1997;81:748-54.

33. Suit HD, Goitein M, Tepper J, Koehler AM, Schmidt RA, Schneider R. Explorotory study of proton radiation therapy using large field techniques and fractionated dose schedules. Cancer. 1975;35:1646-57.

34. Gragoudas ES, Seddon JM, Egan KM, Glynn RJ, Goitein M, Munzenrider J, et al. Metastasis from uveal melanoma after proton beam irradiation. Ophthalmology. 1988;95:992-9.

35. Bedikian AY, Legha SS, Mavligit G, Carrasco CH, Khorana S, Plager C, et al. Treatment of uveal melanoma metastatic to the liver: a review of the M. D. Anderson Cancer Center experience and prognostic factors. Cancer. 1995;76:1665-70.

36. Berry JL, Dandapani SV, Stevanovic M, Lee TC, Astrahan M, Murphree AL, et al. Outcomes of choroidal melanomas treated with eye physics: a 20-year review. JAMA Ophthalmol. 2013; 131:1435-42.

37. Kujala E, Makitie T, Kivela T. Very long-term prognosis of patients with malignant uveal melanoma. Invest Ophthalmol Vis Sci. 2003;44:4651-9. 